\title{
Endoplasmic reticulum stress may be involved in insulin resistance and lipid metabolism disorders of the white adipose tissues induced by high-fat diet containing industrial trans-fatty acids
}

This article was published in the following Dove Press journal:

Diabetes, Metabolic Syndrome and Obesity: Targets and Therapy

\author{
Wanqiu Zhu' ${ }^{1, *}$ \\ Xin Niu ${ }^{\mathrm{I}} *$ \\ Mingxia Wang' \\ Zhao $\mathrm{Li}^{2}$ \\ Hong-Kun Jiang ${ }^{3}$ \\ Chuntao $\mathrm{Li}^{4}$ \\ Samantha J Caton ${ }^{5}$ \\ Yinglong Bai' \\ 'Department of Maternal and Child \\ Health, School of Public Health, China \\ Medical University, Shenyang, Liaoning, \\ People's Republic of China; ${ }^{2}$ Department \\ of Cardiology, The First Hospital of China \\ Medical University, Shenyang, Liaoning, \\ People's Republic of China; ${ }^{3}$ Department \\ of Pediatrics, The First Hospital of China \\ Medical University, Shenyang, Liaoning, \\ People's Republic of China; ${ }^{4}$ Information \\ Center, the First Hospital of China \\ Medical University, Shenyang, Liaoning, \\ People's Republic of China; ${ }^{5}$ School of \\ Health and Related Research (ScHARR), \\ Public Health, University of Sheffield, \\ Sheffield, SI 4DA, UK \\ *These authors contributed equally to \\ this work
}

\begin{abstract}
Background: Consumption of industrially produced trans-fatty acids (iTFAs) can result in alteration to lipid profile and glucose metabolism. Moreover, a diet high in iTFAs could increase the risk of obesity, cardiovascular diseases (CVDs) and type 2 diabetes mellitus. Glucose and lipid metabolism are closely linked in white adipose tissue (WAT), yet the underlying mechanisms of the effect of iTFAs in WAT are poorly understood.

Materials and methods: Parameters of glucose homeostasis, lipid profiles and markers of endoplasmic reticulum (ER) stress of WAT were measured in rats maintained on a high-fat diet containing margarine (HFD-M) $(n=10)$ compared to controls maintained on standard chow $(\mathrm{n}=10)$ over 16 weeks.
\end{abstract}

Results: Fat mass and body weight was significantly increased in rats maintained on the HFD-M compared to controls $(P<0.01)$. HFD-M rats had increased levels of insulin (INS), homeostasis model assessment of insulin resistance and serum lipid profile was significantly altered. The expression of glucose-regulated protein 78 (GRP78) and the phosphorylation of inositol-requiring enzyme 1-alpha and c-Jun N-terminal kinase (JNK) were significantly increased in subcutaneous and retroperitoneal adipose depots of HFD-M-fed rats. In vitro, wider ER lumens were observed in $100 \mu \mathrm{mol} / \mathrm{L}$ elaidic acid (EA)-treated human mature adipocytes. We observed activation of ER stress markers, impaired INS receptor signaling and increased lipogenesis in adipocytes after EA exposure. These effects could be alleviated by inhibiting ER stress in adipocytes in vitro.

Conclusion: Collectively these data suggest that ER stress may be involved in INS resistance and lipid metabolism disorders induced by high-fat diet containing iTFAs. These findings suggest that WAT could be regarded as a key target organ for inhibiting ER stress to reverse the impaired INS receptor signaling, alleviate lipid metabolism disorders, and provide a novel approach to prevent and treat INS resistance and dyslipidemia-related chronic diseases such as T2MD and CVDs.

Keywords: ER stress, elaidic acid, obesity, insulin resistance, dyslipidemia, adipocytes

\section{Introduction}

Despite recent successful attempts at reducing intake of trans-fatty acids in many countries, ${ }^{1}$ industrially produced trans-fatty acids (iTFAs) are still present in a number of manufactured foods which contain partially hydrogenated oils or their products added such as margarine, snack foods, and packaged baked goods. Highfat diets containing iTFAs have received attention due to their potential impact on
Correspondence: Yinglong Bai 年 Health, School of Public Health, China Shenyang North New Area, Shenyan I 10122, Liaoning, People's Republic of China

Tel+86 2431939406

Fax +862431939406

Email ylbai@cmu.edu.cn 
health outcomes associated with alterations to lipid profiles and glucose homeostasis. ${ }^{2}$ Diets high in iTFAs can result in higher rates of obesity ${ }^{3}$ and are associated with chronic diseases, such as cardiovascular diseases (CVDs) and type 2 diabetes mellitus (T2DM). ${ }^{4,5}$

Disorders of glucose homeostasis and lipid metabolism are commonly seen in CVDs and T2DM. Lipid profile abnormalities including representative markers such as triglyceride (TG), high-density lipoprotein-cholesterol (HDL-C), and low-density lipoprotein-cholesterol (LDL-C), are important indicators of cardiac metabolic risk and play a significant role in the development of CVDs. ${ }^{6}$ Insulin (INS) resistance is the main etiology and pathogenesis of T2DM. ${ }^{7}$ Individuals with obesity (excessive white adipose tissue (WAT)) have increased risk of CVDs and T2DM. ${ }^{8,9}$ WAT is the major metabolic organ responsible for energy utilization and storage, in which glucose and lipid metabolism are closely linked. Yet, the underlying mechanisms of the effect of iTFAs on WAT remain poorly understood.

Recent studies suggest that ER stress links obesity, CVDs, INS action, and T2DM. ER stress has also been demonstrated to be a novel mechanism and therapeutic target for CVDs. ${ }^{10}$ ER stress is a major contributor to the development of INS resistance and diabetes by triggering JNK activity via inositol-requiring enzyme 1-alpha (IRE1- $\alpha$ ) and inhibition of INS receptor signaling pathways. ${ }^{11}$ Multiple markers of ER stress have been observed to be activated in human adipose with obesity. ${ }^{12}$ It has been demonstrated that ER stress is associated with de novo lipogenesis and lipid accumulation in HepG2 cells ${ }^{13}$ and INS resistance in B cells in vitro. ${ }^{14}$

The aim of this work is to examine the underlying mechanisms of INS resistance and lipid metabolism disorders of WAT induced by high-fat diets containing iTFAs. In vivo, we measured the parameters of lipid profile and glucose homeostasis and markers of ER stress in subcutaneous and retroperitoneal adipose tissue of rats fed with HFD-M. In vitro, human mature adipocytes were treated with EA, known as a biomarker of iTFAs intake, ${ }^{15}$ to observe the effects on INS receptor signaling and lipogenesis.

\section{Materials and methods}

\section{Animals and diets}

Twenty healthy, SPF-grade-weaned male Wistar rats, were obtained from the Center for Experimental Animals at China Medical University (Shenyang, China) with a National Animal Use License number of SCXK 2013-
0001. All experiments were approved by the Animal Use and Care Committee at China Medical University with a protocol number of CMU 62043006 and in accordance with the National Institutes of Health Guide for the Care and Use of Laboratory Animals.

All rats were randomly divided into two groups: the control group $(\mathrm{CON}, \mathrm{n}=10)$ and the treated group fed with high-fat diets containing margarine (HFD-M, $n=10$ ). Rats were maintained on their respective diets for 16 weeks. The rats in CON group were given standard chow containing $10 \%$ of kcal from fat. And the HFD-M rats were given homemade high-margarine chow as described before, ${ }^{16}$ which contained $45 \%$ of kcal from fat and were composed of $73 \%$ standard chow diet plus $20 \%$ margarine $(1.5 \%$ iTFAs in margarine according to the manufacture's indicated nutrition facts, purchased from a local supermarket), 7\% casein (01-003, Aoboxing Biotech Company Ltd, Beijing, China), and trace amounts of multiple vitamins. All animals were housed at $21-24^{\circ} \mathrm{C}$, with $55-60 \%$ humidity, $12-\mathrm{hr}$ light/dark cycle, and free access to food and water. There was no difference in initial start weights between groups.

\section{Processing of serum and tissues}

At the end of the 16th week, all experimental animals were sacrificed under anesthesia with carbon dioxide after $12 \mathrm{hrs}$ of fasting. Fasting blood samples were drawn via the abdominal aorta for the separation of serum. The subcutaneous and retroperitoneal fat tissues were dissected and weighed in grams and expressed as a percentage of total body weight. Retroperitoneal fat mass (\% of total body weight) $=$ the weight of retroperitoneal WAT $(\mathrm{g}) /$ body weight $(\mathrm{g}) \times 100{ }^{17}$ Tissues were stored at $-80^{\circ} \mathrm{C}$ immediately for the following analysis.

\section{Measurements of INS resistance and lipid profile markers}

Markers of lipid profiles and INS resistance were measured using commercially available kits. Triglycerides (TG, catalog no. 6030), total cholesterol (TCHO, catalog no. Y014), highdensity lipoprotein-cholesterol (HDL-C, catalog no. 6328), low-density lipoprotein-cholesterol (LDL-C, catalog no. 6340), glucose (GLU, catalog no. Y012) (Beijing BHKT Clinical Reagent Co., Ltd) and INS (IQR-Insulin-1, RayBiotech, Inc., China). Homeostasis model assessment of insulin resistance(HOMA-IR) was determined by the formula: HOMA-IR $=$ serum insulin $(\mathrm{mIU} / \mathrm{L}) \times($ blood glucose $(\mathrm{mmol} / \mathrm{L}) / 22.5$. 


\section{Cell culture and treatments}

Human mesenchymal stem cells were obtained from Cyagen Biosciences Inc. (HUXMD-01001) and maintained in DMEM/Nutrient Mixture F-12 Ham (DMEM/ F-12) containing $10 \%$ heat-inactivated FBS and $1 \%$ penicillin/streptomycin. The cells were maintained at $37^{\circ} \mathrm{C}$ in a humidified $5 \% \mathrm{CO}_{2}$ atmosphere. The process of differentiation was operated as described by Wang. ${ }^{16}$ After 14 days of differentiation, mature adipocytes were treated with EA $(100 \mu \mathrm{mol} / \mathrm{L}$; E4637, Sigma, USA) for $24 \mathrm{hrs}$. Transmission electron microscope was then used to observe the cells' ultrastructure. For the following tests of glucose intake, TG, relative quantitative real-time and Western-blot analyses, $10 \mathrm{mmol} / \mathrm{L}$ 4-PBA (SML0309, Sigma, USA) was preincubated for 2 hrs. After EA exposure for $24 \mathrm{hrs}$, the cells were collected.

\section{Transmission electron microscopy analysis}

The cells fixed with $2.5 \%$ glutaraldehyde were post-fixed in $1 \%$ osmium tetroxide, dehydrated through graded ethanol series, and embedded in Spurr's resin. Sections were stained with uranyl acetate and lead citrate. Ultrastructural changes were examined using transmission electron microscope (JEM-1200EX, Hitachi Ltd., Tokyo, Japan). ER luminal diameter was analyzed as previously described, using ImageJ2x software (Version 2.1.4.7, Wayne Rasband, National Institutes of Health, USA) on six electron micrographs taken at $12,000 \times$ magnification from three independent studies. ${ }^{18}$

\section{Cellular glucose uptake and TG measurements}

Cellular glucose uptake was assessed using 2-NBDG (11046, Cayman, USA). The screening system was performed as previously described, with some modifications. ${ }^{19}$ Briefly, the cells were seeded in 96-well flat-bottomed plates with $1 \times 10^{4}$ cells /well in $100 \mu \mathrm{L}$ culture medium. After exposure to $100 \mu \mathrm{mol} / \mathrm{L}$ EA for $24 \mathrm{hrs}$, the adipocytes were starved for $1 \mathrm{hr}$ in glucose-free media. Starved adipocytes were washed three times with PBS and then preincubated with INS (10 nmol/L, 91077C, Sigma, USA) for 15 mins. All cells were then treated for $1 \mathrm{~h}$ with serum-free media containing $150 \mu \mathrm{g} / \mathrm{mL}$ of the 2-NBDG. After $1 \mathrm{hr}$, cells were washed with PBS and the fluorescence was read at wavelength 475/ $550 \mathrm{~nm}$ as instructed by the manufacturer. The level of TG was measured using Triglyceride kit (A110-1, Nanjing Jiancheng Bioengineering Institute, China).

\section{Relative quantitative real-time RT-PCR}

The cells were lysed in Trizol $^{\circledR}$ Reagent $(15,596,018$, Invitrogen, Carlsbad, CA, USA) and RNA was purified according to the manufacturer's instructions. Total RNA was subjected to a reverse transcription reaction using Prime Script RT reagent Kit With gDNA Eraser (Perfect Real Time) (RR047A, TaKaRa, Dalian, China). Random primers were obtained from (DINGGUO, Beijing, China). The following PCR primers were used for real-time: sterol regulatory element-binding protein (SREBP-1c, forward: CTTCCGCCCTTGAGCTG, reverse: CTGGTGTGTCCG TGTGG) and GAPDH (forward: GGATGATGTTCTGG AGAGCC3, reverse: CATCACCATCTTCCAGGAGC).

The generated cDNA was carried out using a SYBR green PCR kit (RR820A, TaKaRa, Dalian, China) on the 7500 Real-Time PCR system (Becton, Dickinson and Company, USA), and the relative gene expression was calculated by the $2^{-\triangle \Delta C T}$ method after normalization to normalized to internal control GAPDH.

\section{Western blot analyses}

The cells were lysed in ice-cold lysis buffer. Samples with approximately $30 \mu \mathrm{g}$ of proteins were electrophoresed in $8 \%$ SDS-PAGE gels, followed by transfer to polyvinylidene difluoride membranes $(0.4 \mu \mathrm{m})$ and blocking with $5 \%$ BSA for $1 \mathrm{hr}$. After blocking, the membranes were incubated overnight with FAS (1:1000 dilution, 3180S, Cell Signaling Technology, USA), GRP78 (1:1000 dilution, 3183S, Cell Signaling Technology, USA) IRE1- $\alpha$ (1:1000 dilution, 3294S, Cell Signaling Technology, USA), p-IRE1- $\alpha$ (1:1000 dilution, ab124945, Abcam, UK), JNK (1:1000 dilution; 9252S, Cell Signaling Technology, USA), p-JNK (1:1000 dilution, 4668S, Cell Signaling Technology, USA), IRS-1 (1:1000 dilution, 2382S, Cell Signaling Technology, USA), p-IRS-1 (Ser307) (1:1000 dilution, 2381S, Cell Signaling Technology, USA), GLUT4 (1:1000 dilution, A7637, ABclonal, China), GAPDH (1:1000 dilution; 5174S, Cell Signaling Technology, USA) at $4^{\circ} \mathrm{C}$. Then, peroxidase-conjugated goat anti-rabbit IgG (1:5000 dilution, ZB-5301, Beijing Zhongshan Golden Bridge Biotechnology Co, China) was used for $1 \mathrm{hr}$ at room temperature. Protein bands were detected by using ECL Western blotting chemiluminescent detection reagents (170-5060, BIO-RAD, USA) and then quantified by Image $2 \mathrm{x}$ software (Version 2.1.4.7, Wayne Rasband, National Institutes of Health, USA). GAPDH was used as the internal standard to normalize the signals. 


\section{Statistical analysis}

Statistical analyses were performed with SPSS software (version 19.0, SPSS Inc., Chicago, IL, USA). The data are expressed as mean $\pm \mathrm{SD}$. Student's $t$-test and one-way ANOVA were carried out, and significance was indicated at $P<0.05$.

\section{Results}

\section{Body weight}

After 16-week feeding, rats treated with high-fat diets containing margarine (HFD-M) gained significantly more weight than those fed with standard chow (Figure 1A and B). Similarly, rats maintained on the HFD-M group accumulated significantly more retroperitoneal fat (total (g) and \% of total body weight) compared to $\mathrm{CON}$ rats (Figure 1C and D).

\section{Insulin resistance and serum lipids}

The data are shown in Table 1. No significant differences were observed in fasting GLU level between the

\section{A}
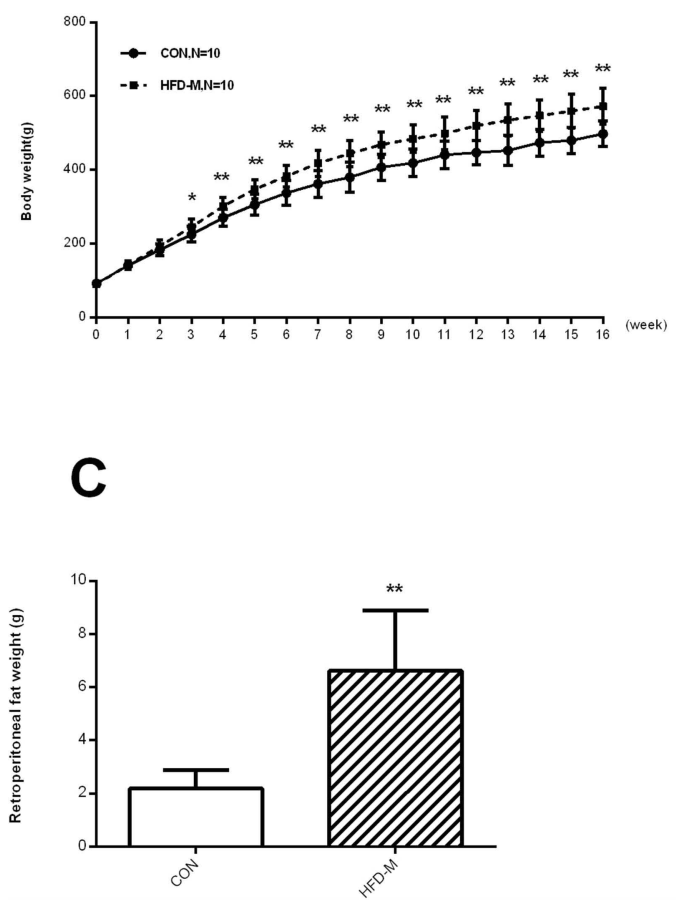

CON and HFD-M groups. Compared with the CON group, the levels of INS, HOMA-IR, TG, TCHO, LDL-C, and HDL-C in HFD-M group were significantly increased.

\section{Markers of ER stress in subcutaneous and retroperitoneal adipose tissue}

Compared with the CON group, the expression level of GRP78, known as an ER stress response indicator, was markedly higher in HFD-M group. The same patterns of change were observed for IRE1- $\alpha$ phosphorylation and JNK phosphorylation, indicating that margarine feeding caused ER stress in subcutaneous (Figure 2A-D) and retroperitoneal (Figure 3A-D) fat tissues of rats.

\section{The effects of EA on the ultrastructure in human mature adipocytes in vitro}

Transmission electron microscope analysis of the ultrastructure of the human mature adipocytes is shown in Figure 4. The adipocytes exhibited normal integral

B
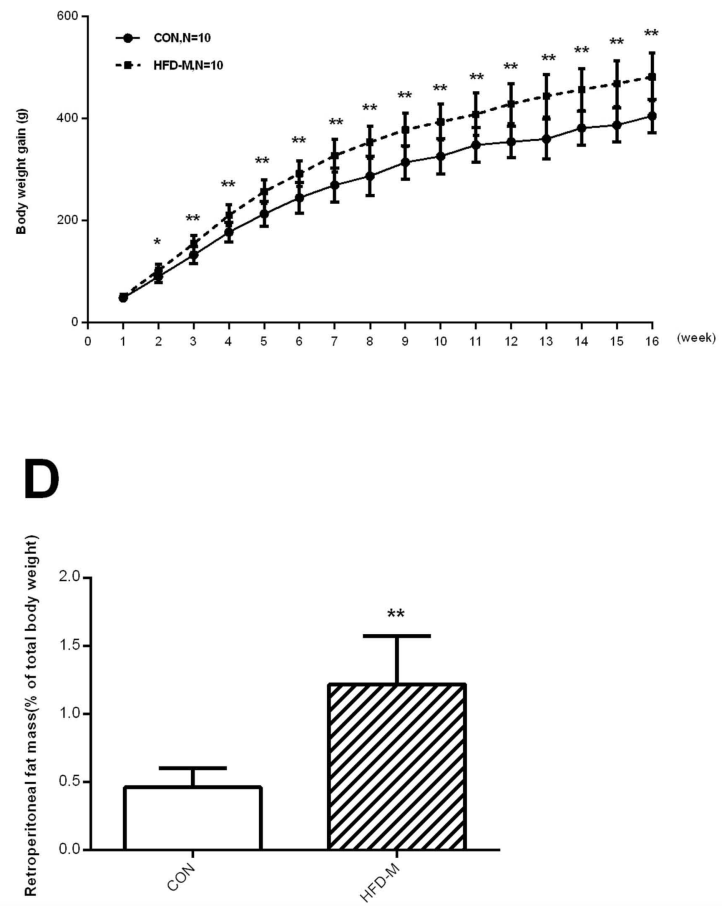

Figure I Body weight and retroperitoneal fat mass in control $(n=10)$ and HFD-M $(n=10)$ rats. The comparison between CON and HFD-M groups in (A) Body weight; (B) body weight gain; (C)retroperitoneal fat weight; (D) retroperitoneal fat mass (\% of total body weight). $* P<0.05$, $* * P<0.0$ I.

Abbreviations: CON, control; HFD-M, high-fat diets containing margarine. 
Table I The comparison of serum glucose and lipid metabolism measurements (mean $\pm S D, n=10$ )

\begin{tabular}{|l|l|l|}
\hline & CON & HFD-M \\
\hline GLU (mmol/L) & $10.16 \pm 2.72$ & $11.10 \pm 1.03$ \\
INS (mlU/L) & $17.85 \pm 8.55$ & $28.82 \pm 10.54^{*}$ \\
HOMA-IR & $8.56 \pm 5.66$ & $14.26 \pm 5.42^{*}$ \\
TG (mmol/L) & $0.27 \pm 0.04$ & $0.33 \pm 0.05^{* *}$ \\
TCHO (mmol) & $0.94 \pm 0.13$ & $1.48 \pm 0.31^{* *}$ \\
LDL-C (mmol/L) & $0.61 \pm 0.12$ & $1.01 \pm 0.24^{* *}$ \\
HDL-C (mmol/L) & $0.58 \pm 0.11$ & $0.70 \pm 0.19^{* *}$ \\
\hline
\end{tabular}

Notes: $* P<0.05$, $* * P<0.01$, compared with $C O N$ group using an independent groups Student's $t$-test.

Abbreviations: CON, control; HFD-M, high-fat diets containing margarine; GLU, glucose; INS, insulin; HOMA-IR, homeostasis model assessment of insulin resistance; TG, triglyceride; TCHO, total cholesterol; LDL-C, triglyceride; HDL-C, high-density lipoprotein-cholesterol.

structures, the central nucleus, the well-developed organelles, and the presence of small lipid droplets (Figure 4A and B). Compared with the control, the width of the ER lumens in the treated group was significantly larger (Figure 4C), indicating that ER stress may occur after exposure to $100 \mu \mathrm{mol} / \mathrm{L}$ EA for $24 \mathrm{hr}$ in human mature adipocytes.

\section{Activated ER stress markers in mature adipocytes exposed by EA}

In vitro, indications of ER stress were tested in human mature adipocytes. Compared with the control group, the expression of ER stress-related protein GRP78, IRE1- $\alpha$, and JNK phosphorylation in EA exposure group was significantly increased (Figure 5A-D). These results suggested that ER stress occurred after exposure to $100 \mu \mathrm{mol} / \mathrm{L}$ EA for $24 \mathrm{hrs}$ in human mature adipocytes. Meanwhile, the administration of 4-phenylbutyric acid (4-PBA) reduced GRP78 expression and the

B

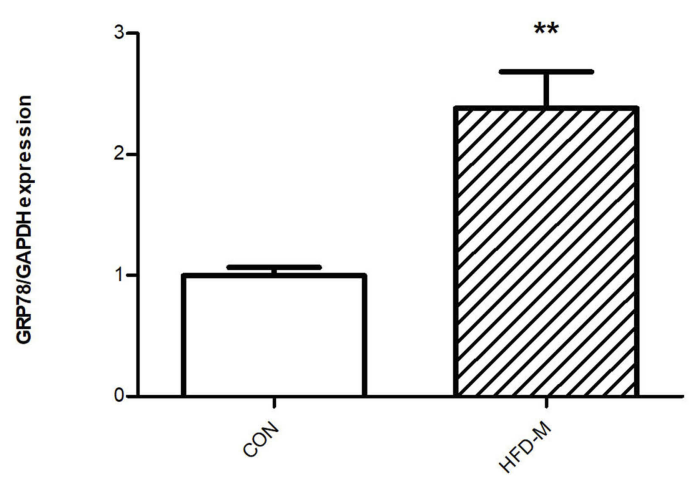

C

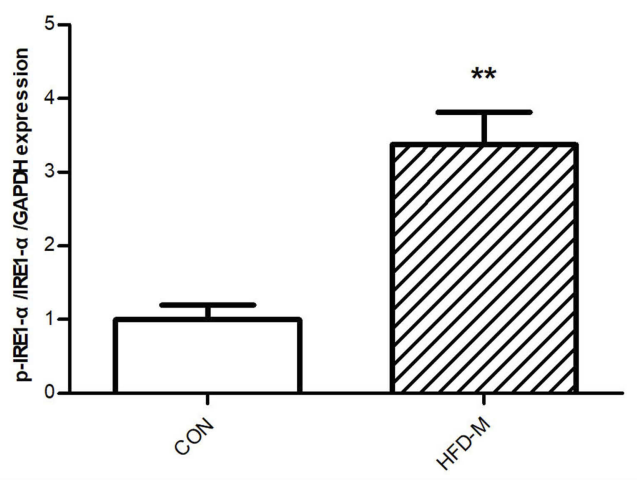

D

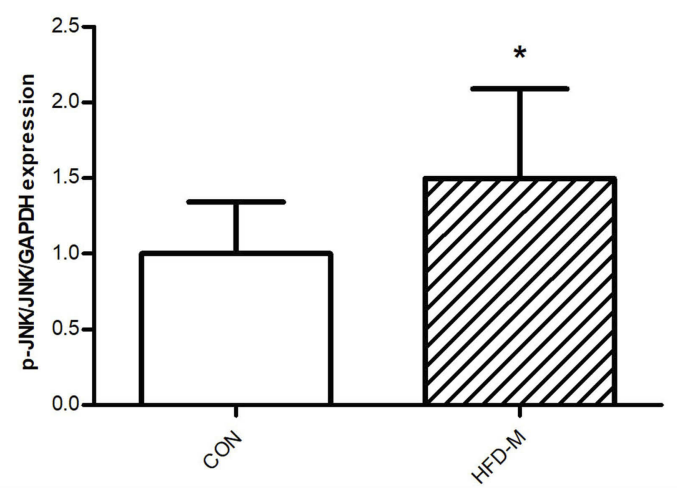

Figure 2 Effects of HFD-M on the expression of ER stress-related proteins in subcutaneous fat tissue of rats. (A) Representative GRP78, p-IREI-a, P-JNK Western blots;

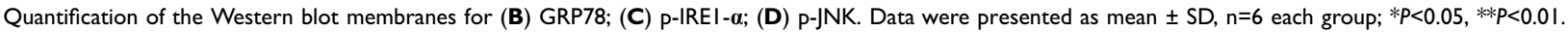

Abbreviations: ER, endoplasmic reticulum; GRP78, glucose-regulated protein 78; p-IREI- $\alpha$, phosphorylated Inositol-requiring enzyme I-alpha; p-JNK, phosphorylated c-Jun n-terminal kinase. 
A

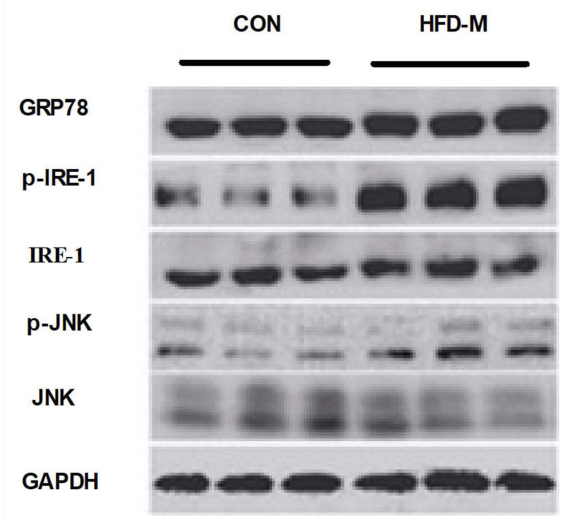

C

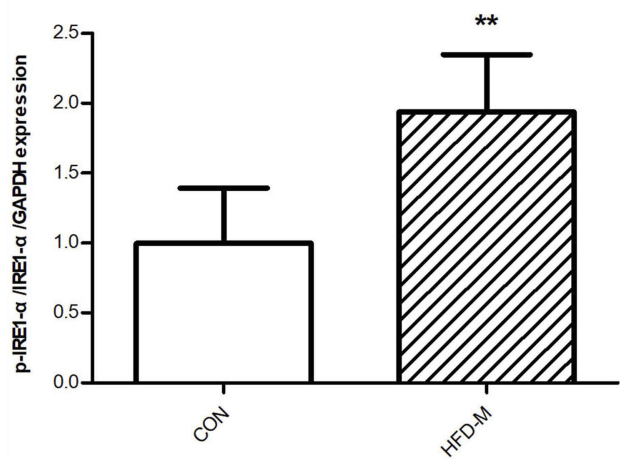

B

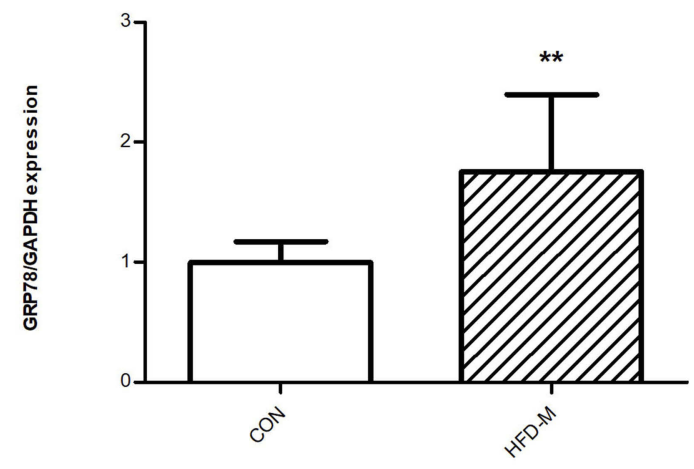

D

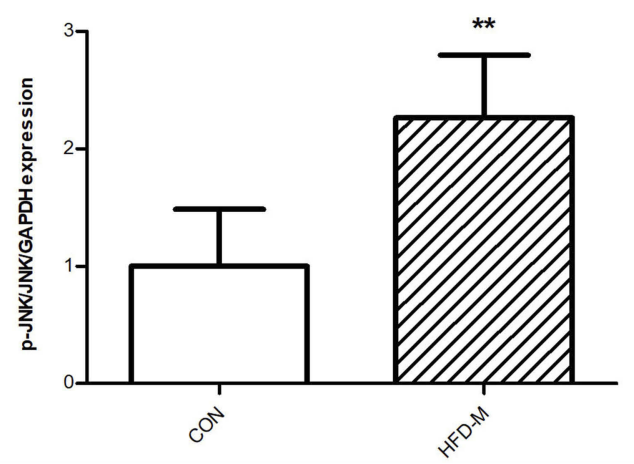

Figure 3 Effects of HFD-M on the expression of ER stress-related proteins in rat retroperitoneal fat tissue. (A) Representative GRP78, P-IREI- $\boldsymbol{\alpha}$, P-JNK Western blots;

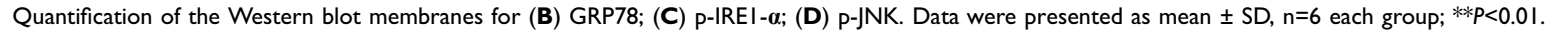

Abbreviations: ER, endoplasmic reticulum; GRP78, glucose-regulated protein 78; p-IREI- $\alpha$, phosphorylated Inositol-requiring enzyme I-alpha; p-JNK, phosphorylated c-Jun n-terminal kinase.

phosphorylation of IRE1- $\alpha$ and JNK, which indicated that 4-PBA reversed ER stress in mature adipocytes.

\section{ER stress involves in impaired INS receptor signaling induced by EA in mature adipocytes}

Capacity for glucose uptake was assessed. No significant difference was seen between the treated groups (Figure 6A). Changes in glucose transporter 4 (GLUT4) expression were not obvious (Figure $6 \mathrm{~B}$ and $\mathrm{C}$ ). However, the expression of serine phosphorylated INS receptor substrate 1 (p-IRS-1) was significantly increased by EA exposure (Figure 6D). Additionally, the administration of 4-PBA reduced p-IRS-1(ser) level in mature adipocytes.

\section{ER stress involves in fat accumulation of adipocytes induced by EA}

Compared with the control group, TG level increased after exposure to $100 \mu \mathrm{mol} / \mathrm{L}$ EA for $24 \mathrm{hrs}$ in human mature adipocytes (Figure 7A). Compared with the EA group, cells preincubated with 4-PBA have significantly lower TG content (Figure 7A). The relative expression of SREBP-1c mRNA was upregulated after EA exposure and could be downregulated by 4-PBA administration (Figure 7B). Compared with the control group, the expression of fatty acid synthase (FAS) was increased significantly by EA exposure and could be effectively reduced by 4-PBA administration (Figure 7C and D). 

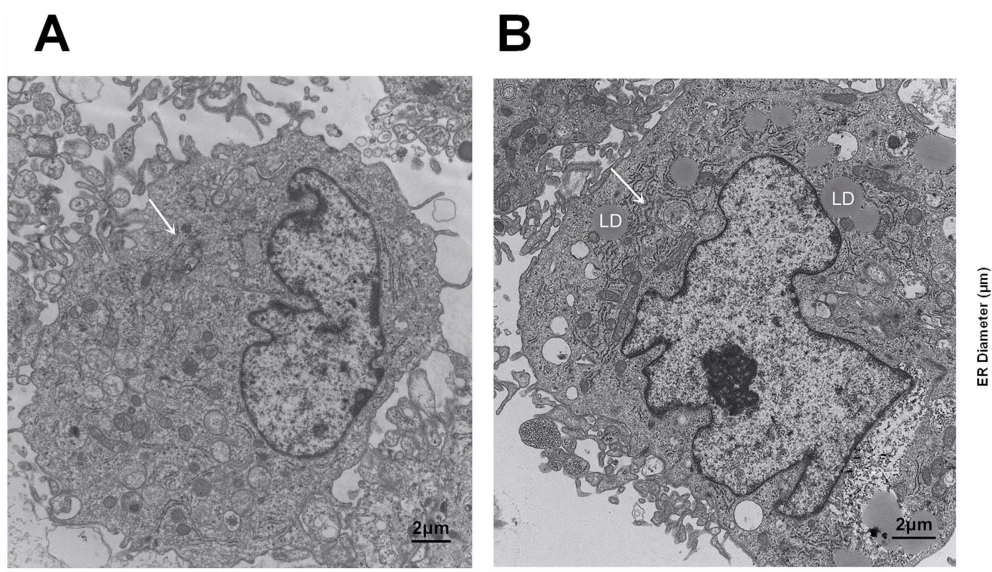

\section{C}

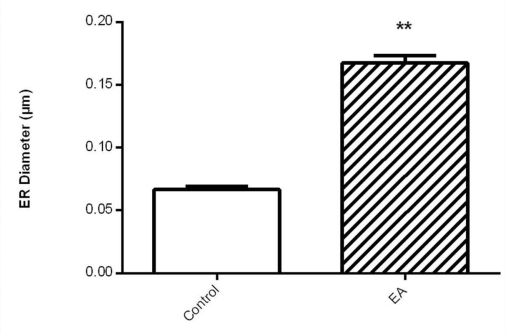

Figure 4 ER stress induced by EA in human mature adipocytes. (A) Control group; (B) treated with $100 \mu$ mol/L of EA for 24 hrs; (C) quantitative analysis of ER luminal diameter, as a surrogate marker for ER stress per cell using transmission electron microscope images. Results are representative averages of at least six images performed from three independent experiments and are displayed as mean $\pm S D$. $* * P<0.0$ I. White arrow shows the ER lumen.

Abbreviations: ER, endoplasmic reticulum; EA, elaidic acid; LD, lipid droplets.

\section{Discussion}

The aim of the current research was to examine the underlying mechanisms of INS resistance and lipid metabolism disorders of WAT induced by high-fat diets containing iTFAs. In line with previous research on saturated fatty acids rich diets, ${ }^{16,20}$ we observed that the rats maintained for 16 weeks on the HFD-M gained significantly more body weight and retroperitoneal fat mass compared to controls. Retroperitoneal fat is an active endocrine organ that is associated with the development of INS resistance and dyslipidemia in patients with metabolic syndrome. ${ }^{21}$ HFD-M rats demonstrated significantly increased INS, HOMA-IR, TG, TCHO, LDL-C, and HDL-C compared to controls. Additionally, in the HFD-M-maintained rats, increased expression of GRP78 and the phosphorylation of IREI- $\alpha$ and JNK were significantly elevated. Together, these findings indicate the potential for diets high in ITFAs to induce positive energy balance and ER stress in rodents. Further, human adipocytes exposed to EA for $24 \mathrm{hrs}$ demonstrated increased ER lumens width and expression of ER stress markers. Interestingly, ER stress was reversed by 4-PBA suggesting a potential therapeutic effect.

In the current study, INS resistance occurred in the rats fed with HFD-M. Fasting GLU, INS, and HOMA-IR are acknowledged as effective indicators associated with glucose metabolism disorder. As we reported previously, ${ }^{16}$ INS levels and HOMA-IR were increased significantly after the 16-week HFD-M regimen. Our findings support previous animal studies demonstrating that high-TFA diets induce insulin resistance in rodents ${ }^{22}$ and results in impaired INS sensitivity in monkeys. ${ }^{23}$ Previous work on humans also demonstrates that increased iTFAs consumption is associated with INS resistance and lipid metabolism disorder. ${ }^{24,25}$ A survey carried out in US adults reported that higher plasma concentrations of total TFAs were associated with T2DM. ${ }^{5}$

TFAs are known to affect lipid metabolism. In recent years, enough evidence has been accumulated to reveal the unfavorable effects of dietary iTFAs on lipid profiles. ${ }^{26} \mathrm{EA}$ is widely used as a specific biomarker of margarine intake in several investigations and can sufficiently reflect the bioavailability and the subsequent harmful effects of margarine or iTFAs. ${ }^{27}$ Data from human studies have demonstrated a positive relationship between blood EA level and lipid parameters, such as LDL-C and TG. ${ }^{28}$ High iTFAs intake is detrimental to cardiovascular health, ${ }^{29}$ and a strong correlation between high dietary intake of iTFAs and a high risk of CVDs has been consistently demonstrated. ${ }^{30}$ Lipid profile including TCHO, LDL-C, and TG are routine biochemical tests used to predict the risk for lipid metabolism disorder. In the present study, the HFD-M rats had increased concentrations of serum TG, TCHO, LDL-C, and HDL-C. Similar results were reported in mouse models (Hwang et al) where rodents fed HFD-M also demonstrated elevated HDL-C and LDL-C levels. ${ }^{31}$ HDL consists of a heterogeneous group of particles with major differences in their structural, biological and functional properties. ${ }^{32}$ Earlier studies have indicated that higher HDL-C level is beneficial. However, more recent evidence has challenged this idea. It has been suggested that the high HDL-C levels may actually be associated with increased risk for coronary artery 

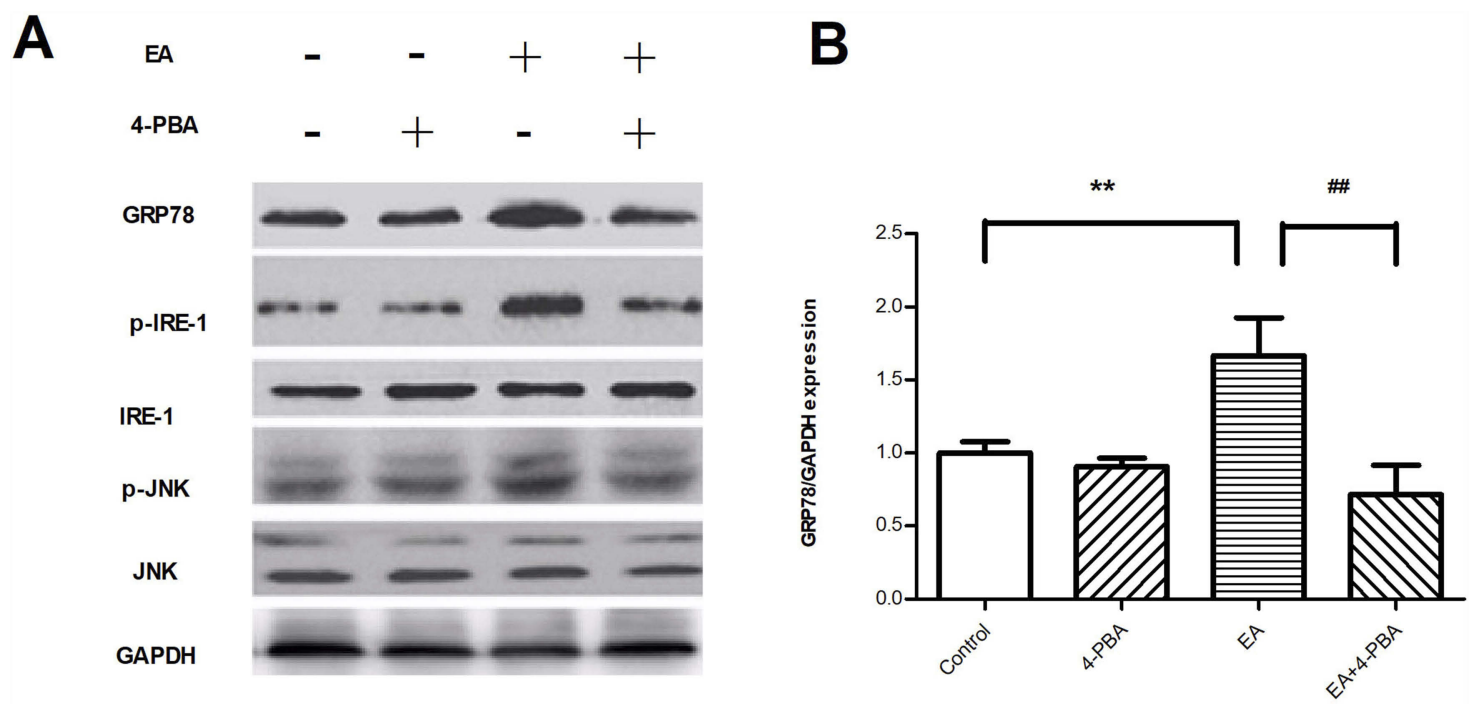

C
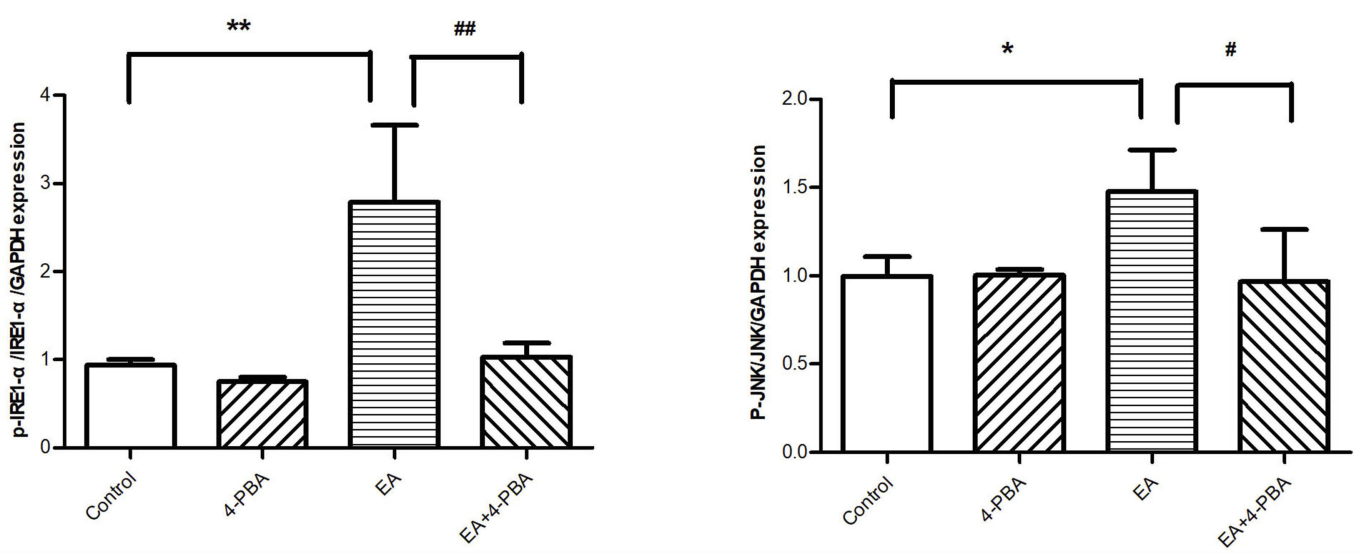

Figure 5 EA exposure activated markers of ER stress pathways in human adipocytes. (A) Representative GRP78, P-IREI- $\alpha$, P-JNK Western blots; quantification of the Western blot membranes for (B) GRP78; (C) P-IREI- $\boldsymbol{\alpha}$; (D) P-JNK. Results are representative averages of at least three independent experiments and displayed as means \pm SD. Compared to control group $* P<0.05$, ${ }^{* * P}<0.01$; compared to EA-treated group, ${ }^{\#} P<0.05,{ }^{\#} P<0.01$.

Abbreviations: ER, endoplasmic reticulum; EA, elaidic acid; 4-PBA, 4-phenylbutyric acid; GRP78, glucose-regulated protein 78; p-IREI- $\alpha$, phosphorylated Inositol-requiring enzyme I-alpha; p-JNK, phosphorylated c-Jun n-terminal kinase.

disease. ${ }^{33}$ Although the current study did not perform further examinations, recent research has indicated that HDL particle subpopulations and HDL functionality play a much more important role in atheroprotection than circulating HDL-C levels. ${ }^{33}$

WAT is currently recognized as an active endocrine organ with complex physiological functions in systemic homeostasis, which also plays an important role in regulating lipid release and storage. ${ }^{34}$ Excessive WAT mass, either in peripheral or in visceral depot, is the consequence of lipid accumulation in white adipocytes, which is the central part of obesity development. ${ }^{35}$ Previous research has suggested that multiple markers of ER stress have been activated not only in the liver ${ }^{36}$ and hippocampus ${ }^{37}$ but also in obese adipose tissues of animals by diet enriched lard. ${ }^{38}$

ER is a membrane-bound organelle in mammalian cells that is responsible for proper folding, processing, and trafficking of proteins. Numerous environmental, physiological, and pathological insults disturb ER homeostasis, known as ER stress, in which a collection of conserved intracellular signaling pathways is activated to maintain ER function for cell survival. ${ }^{15}$ The characteristic of ER stress is that unfolded or misfolded protein retention in the ER causes an unfolded protein reaction (UPR). The UPR is mediated through three ER transmembrane receptors, which are classified as types I and II. IRE1 and doublestranded RNA-activated protein kinase-like endoplasmic 
A

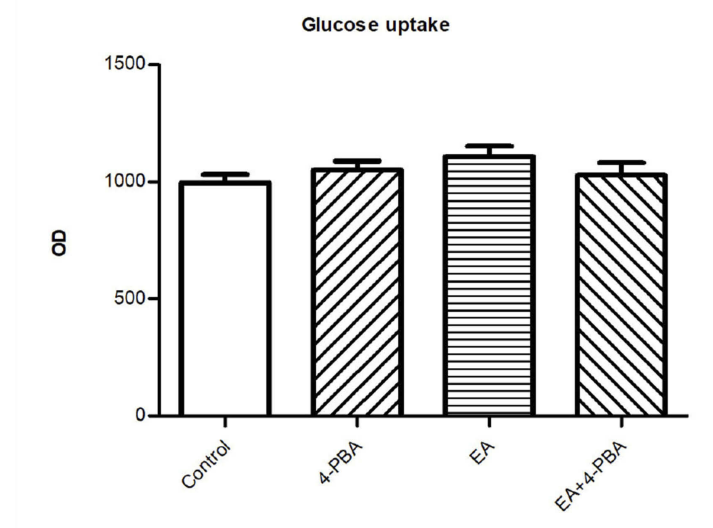

C

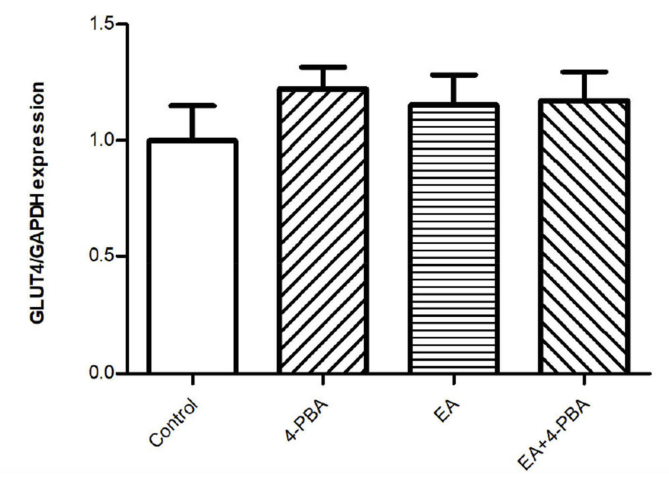

B

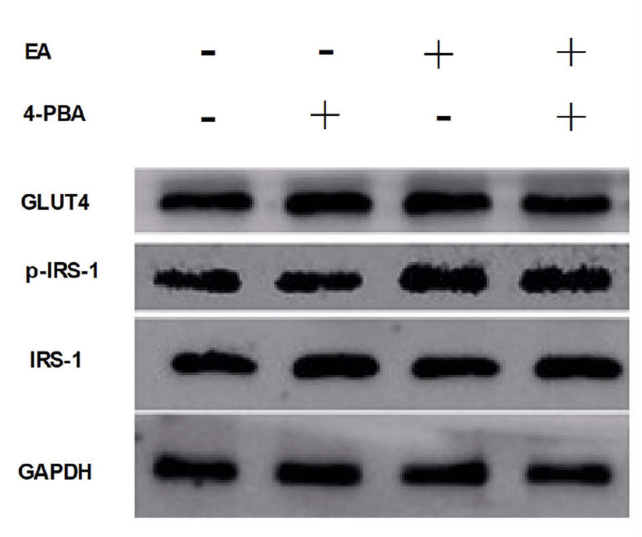

D

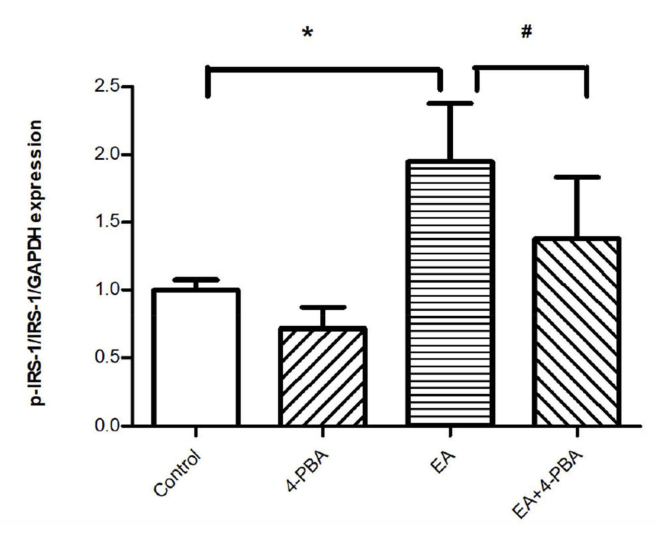

Figure 6 ER stress involves impaired insulin receptor signaling in human adipocytes. (A) Glucose uptake capacity; $(n=6)$; $(\mathbf{B})$ representative Western blots of GLUT4 and IRS-I in human mature adipocytes; (C) quantification of the Western blot for GLUT4; (D) quantification of the Western blot for IRS-I. ( $\mathrm{n}=3$ ); ${ }^{*}<0.05$ compared to control group. ${ }^{\#} P<0.05$ compared to EA group.

Abbreviations: EA, elaidic acid; 4-PBA, 4-phenylbutyric acid; GLUT4, glucose transporter 4; IRS-I, insulin receptor substrate I; P-IRS-I, phosphorylated insulin receptor substrate I.

reticulum kinase are type I proteins possessing protein kinase activities. Activating transcription factor 6 is a type II transmembrane protein encoding a transcription factor, which corresponds to nonfunctional misfolded proteins. ${ }^{39}$ GRP78 is the master of the UPR and can be found in all eukaryotes. ${ }^{40}$ In the standard conditions of balance in the cell, GRP78 is bounded in an inactive form to IRE1 which is a transmembrane stress sensor of UPR. When unfolded or misfolded proteins accumulate in the ER, GRP78 is released from IRE1 to assist with the folding of accumulated proteins, ${ }^{41}$ resulting in activation of $p$-IRE1 and signal transduction to the nucleus. ${ }^{42}$ IRE1 ultimately induces the activation of the JNK pathway. ${ }^{43}$ Previous work has reported the presence of IRE1- $\alpha$ in adipose tissue ${ }^{44}$ and IRE1- $\alpha$ plays a crucial role in INS receptor signaling. ${ }^{38}$ The IRE1-JNK branch activity of the UPR could inhibit INS receptor signaling through direct activation of serine phosphorylation of IRS-1. ${ }^{38,45}$ JNK also plays an important role in lipid metabolism in adipocytes. $^{46,47}$ The current study demonstrates that the expression levels of GRP78 and the phosphorylation of IRE1- $\alpha$ and JNK were significantly increased in HFD-M group. These activated markers indicate that margarine feeding caused UPR to increase ER stress in rat subcutaneous and retroperitoneal fat tissues.

The increase of ER stress-related proteins plays a causal role in the development of obesity-associated metabolic disorders in lean INS-sensitive and obese INS-resistant patients. ${ }^{48}$ ER stress has been regarded as a central feature of peripheral INS resistance and T2DM at 
A

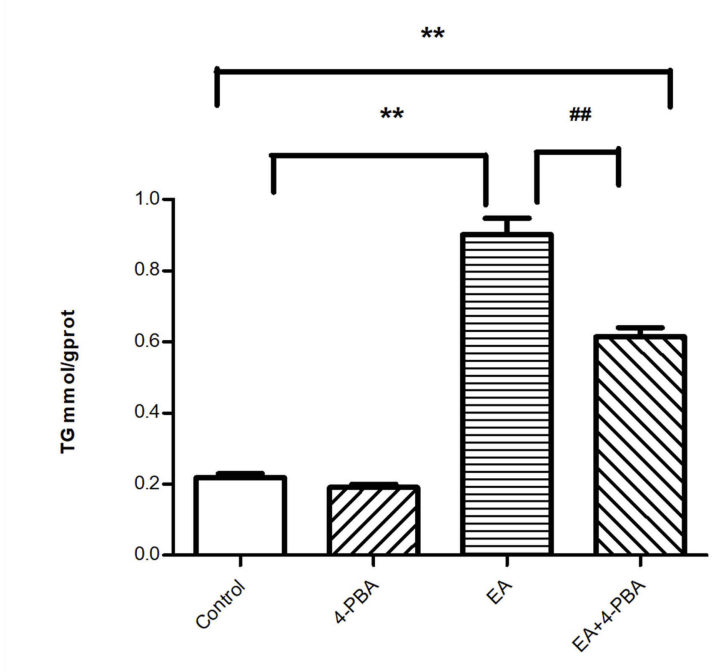

C

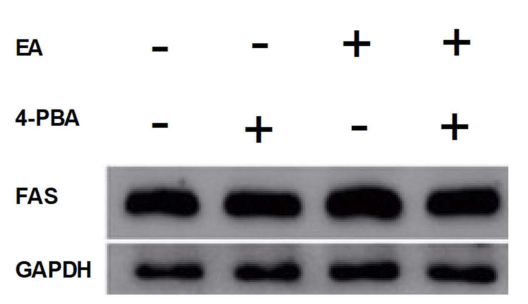

B

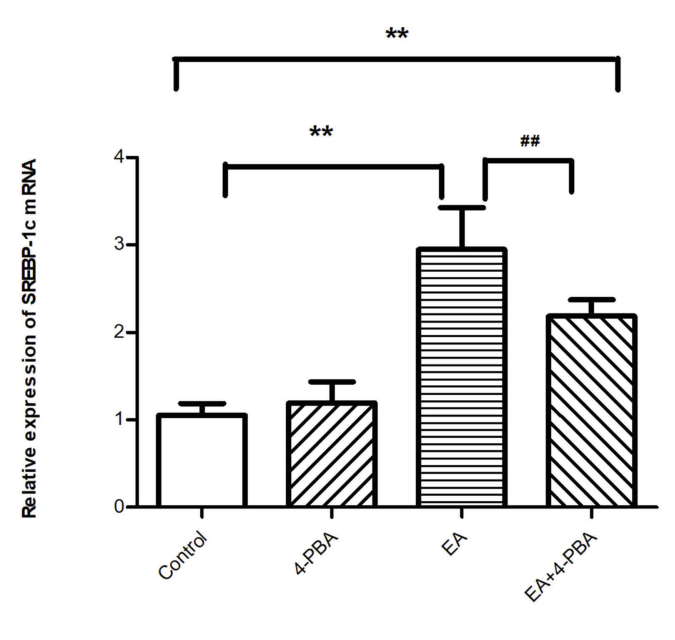

D

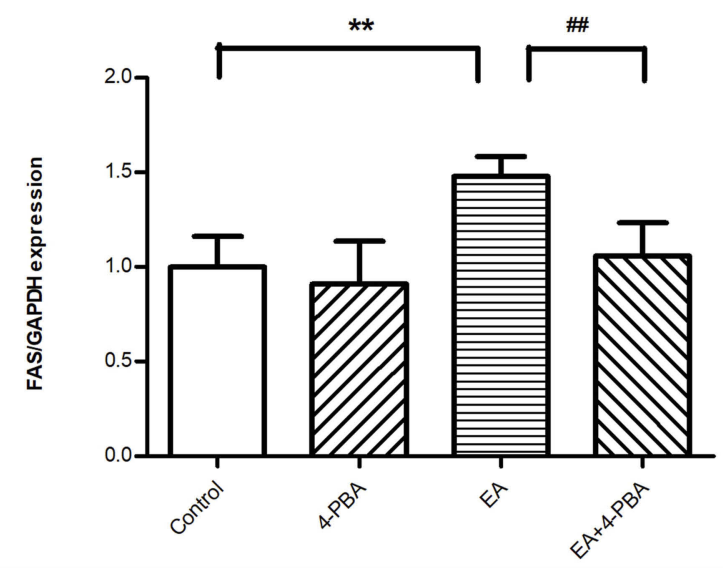

Figure 7 Effects of EA exposure on fat accumulation of human mature adipocytes. (A) TG content. ( $n=6$ ); (B) relative expression of SREBP-Ic mRNA. ( $n=6$ ); (C) representative FAS Western blots. (D) Quantification of the Western blot membranes for FAS. ( $n=3$ ); Data are presented as means \pm SD; Compared to control group $* * P<0.01$; compared to EA group ${ }^{\#} P<0.01$.

Abbreviations: TG, triglyceride; EA, elaidic acid; 4-PBA, 4-phenylbutyric acid; SREBP-Ic, sterol regulatory element binding protein Ic; FAS, fatty acid synthase.

the cellular and organismal levels. ${ }^{38}$ It is also involved in lipid metabolism through modulating the expression levels of key enzymes involved in lipid synthesis or modification. $^{49}$ In the current investigation in vitro, EA, as a typical biomarker of iTFAs, was used to assess whether ER stress involves in INS action and lipid metabolism in differentiated human adipocytes.

The results from the transmission electron microscopy analysis demonstrated that the width of the ER in the treated adipocytes was larger compared to the control. We also demonstrated that expression of ER stress-related protein GRP78, IRE1- $\alpha$, and JNK phosphorylation in the EA exposure group was significantly increased compared to controls. Overall, our results verified that ER stress was induced by EA in human mature adipocytes. 4-PBA is regarded as a chemical or pharmaceutical chaperone which is good at stabilizing protein conformation, improving ER folding capacity and facilitating the trafficking of mutant proteins. ${ }^{50}$ It is used to repress ER stress activation in vitro and in vivo. ${ }^{51,52}$ In our experiment, we found that increased GRP78, IRE1- $\alpha$, and JNK phosphorylation induced by EA could be significantly downregulated after preincubating with 4-PBA, illustrating that ER stress is potentially reversible.

Glucose uptake in peripheral tissues is largely mediated by an intrinsic membrane protein known as facilitative glucose transporters (GLUTs), ${ }^{53}$ and GLUT4 is selectively expressed in INS-sensitive tissues such as 
muscle and adipose cells. ${ }^{54}$ It has been confirmed that the ability of glucose uptake is associated with the expression quantity of cellular GLUT4 in adipose. ${ }^{55}$ Similar to previous reports, we did not observe a prominent increase in glucose uptake ability and the expression of GLUT4 in the presence of EA in the present vitro experiment. ${ }^{56,57}$ These results confirmed that the glucose uptake capacity was functioning properly after EA exposure in mature adipocytes. Moreover, previous research has suggested that the INS-signaling cascade has sufficient plasticity to accommodate significant changes in specific components without further altering glucose uptake. ${ }^{58}$ We explored whether the INS action was altered by EA incubation since adipose tissue is one of the three major target organs of INS. Previous work has verified the role of ER stress in INS resistance in several experimental systems by triggering JNK activity via IRE-1 and inhibition of INS receptor signaling. ${ }^{59,60}$ INS sensitivity can be improved by 4-PBA through reversing ER stress in adipose tissue. ${ }^{61}$ It has been shown that IRS-mediated INS receptor signaling dysregulation is a common underlying mechanism that can produce and exacerbate INS resistance in many genetic and physiological factors. ${ }^{62,63}$ INS signaling in adipocytes is critical for the development of obesity. ${ }^{64}$ ER stress promotes a JNK activity, which in turn inhibits INS receptor signaling through direct activation of serine phosphorylation of IRS- $1 .{ }^{38,45}$ In the present study, we observed that EA exposure could stimulate the serine phosphorylation of IRS-1 via activating ER stress, thereby impairing INS receptor signaling. In addition, impaired GLUT4 translocation to the membrane has also been reported to contribute to INS resistance. ${ }^{65}$ Despite the fact that this was not fully explored in the current study, the ability of glucose uptake was maintained properly. Conflicting results exist for the involvement of IRS-1 in INS-stimulated glucose uptake. Evidence from studies of 3T3 L1 adipocytes as well as adipocytes from non-INS-dependent diabetes mellitus patients suggested that other mechanisms that activate phosphatidylinositol 3-kinase, such as through docking to IRS-2, could play an important role in INS-stimulated adipocytes glucose uptake. ${ }^{66,67}$ Furthermore, researchers observed that superactivation of Rac-1, known as one of the important signaling molecules in cascade activated by INS, could trigger INS-independent GLUT4 translocation via membrane accumulation of $\mathrm{PI}(3,4,5) \mathrm{P} 3$, which results in phosphorylation of Akt and AS160 that rescues INS resistance in muscle cells. ${ }^{68}$
The ER is the major site of lipid metabolism, as many enzymes involved in lipid metabolism are located in the ER. Sterol regulatory element-binding proteins (SREBPs) are transcription factors that belong to the basic helix-loophelix leucine zipper family and are involved in the transcriptional regulation of lipogenic enzymes. ${ }^{69}$ SREBP-1c, an isoform of SREBPs, is expressed in the adipocytes. ${ }^{70}$ Prior reports have suggested that an understanding of SREBP-1c activation in the accumulation of TG appears to be essential. ${ }^{71}$ ER stress can cause SREBP-1c activation. ${ }^{72}$ Ning et al ${ }^{73}$ also observed that phosphorylation of IRE1 $\alpha$ (an upstream activator of X-box-binding protein 1, known as XBP1) and splicing (activation) of XBP1 was elevated in the liver of mice with INS resistance/hyperinsulinemia induced by a high-fat diet; overexpression of the activated XBP1 elevated the promoter activities of the SREBP-1c and FAS genes. Researchers have confirmed that JNK depletion affects basal levels of gene expression involved in lipid metabolism in mouse adipocytes. ${ }^{46}$ And JNK2/SREBP-1c pathway mediates INS-induced fatty acid synthesis in human adipocytes. ${ }^{47}$ We found that the relative expression of SREBP-1c mRNA is significantly upregulated via EA induced ER stress. The expression of FAS, known as SREBP-1c targeting enzymes for de novo fatty acid synthesis, ${ }^{74}$ was also upregulated in adipocytes by EA, and such an effect could be reversed by 4-PBA pre-incubation. Moreover, more TG content was detected in EA-treated group, which indicates that EA promoted lipid accumulation in adipocytes. Collectively, these findings demonstrated that EA exposure activated ER stress, thereby upregulating the expression of lipogenic gene and contributing to lipid accumulation.

\section{Conclusion}

In summary, our results demonstrate the potential mechanism of impaired INS signaling and disorder of lipogenesis induced by iTFAs, which may be due to ER stress activation in WAT. With respect to public health, our data contribute valuable evidence for the importance of healthy food choices and limited intake of iTFAs. In addition, these findings suggested that WAT could be regarded as a key target organ for inhibiting ER stress to alleviate INS resistance and dyslipidemia and provide a new approach to prevent and treat glycolipid metabolism disorders-related chronic diseases such as T2MD and CVDs. 


\section{Acknowledgment}

This research was supported by the National Natural Science Foundation of China (No. 81373018 and 81172691) and Science and Technology Project of Shenyang (No. 19-104-4-002). We are very grateful to Prof. Weidong Zhao for his assistance with transmission electron microscopy analysis.

\section{Disclosure}

The authors declare no conflicts of interest in this work.

\section{References}

1. Wanders AJ, Zock PL, Brouwer IA. Trans fat intake and its dietary sources in general populations worldwide: a systematic review. Nutrients. 2017;9:840. doi:10.3390/nu9080840

2. Mazidi M, Cicero AF, Kengne AP, Banach M. Association between plasma trans-fatty acid concentrations and measures of glucose homeostasis and cardiovascular risk factors in adults in NHANES 1999-2000. Angiology. 2018;69:630-637. doi:10.1177/0003319717745987

3. Chajès V, Biessy C, Ferrari P, et al. Plasma elaidic acid level as biomarker of industrial trans fatty acids and risk of weight change: report from the EPIC study. PLoS One. 2015;10:e118206. doi:10.1371/journal.pone.0118206

4. Wang Q, McKenna M, Borges ÁH, Duprez D, Neuhaus J, Neaton JD. Plasma phospholipid trans-fatty acids levels, cardiovascular diseases, and total mortality: the cardiovascular health study. J Am Heart Assoc. 2014;3. doi:10.1161/JAHA.114.000914.

5. Liu B, Sun Y, Snetselaar LG, et al. Association between plasma transfatty acid concentrations and diabetes in a nationally representative sample of US adults. J Diabetes. 2018;10:653-664. doi:10.1111/ 1753-0407.12652

6. Gomez de la Camara A, De Andrés Esteban E, Urrútia Cuchí G, et al. Variability of nutrients intake, lipid profile and cardiovascular mortality among geographical areas in Spain: the DRECE study. Geospat Health. 2017;12:524. doi:10.4081/gh.2017.524

7. Li C, He JZ, Zhou XD, Xu X. [Berberine regulates type 2 diabetes mellitus related with insulin resistance]. Zhongguo Zhong Yao Za Zhi. 2017;42:2254-2260. doi:10.19540/j.cnki.cjcmm.20170307.014

8. Elagizi A, Kachur S, Lavie CJ, et al. An overview and update on obesity and the obesity paradox in cardiovascular diseases. Prog Cardiovasc Dis. 2018;61:142-150. doi:10.1016/j.pcad.2018.07.003

9. Sonmez A, Yumuk V, Haymana C, et al. Impact of obesity on the metabolic control of type 2 diabetes: results of the Turkish nationwide survey of glycemic and other metabolic parameters of patients with diabetes mellitus (TEMD obesity study). Obes Facts. 2019;12:167-178. doi:10.1159/000496624

10. Liu MQ, Chen Z, Chen LX. Endoplasmic reticulum stress: a novel mechanism and therapeutic target for cardiovascular diseases. Acta Pharmacol Sin. 2016;37:425-443. doi:10.1038/aps.2015.145

11. Flamment M, Hajduch E, Ferre P, Foufelle F. New insights into ER stress-induced insulin resistance. Trends Endocrinol Metab. 2012;23:381-390. doi:10.1016/j.tem.2012.06.003

12. Sharma NK, Das SK, Mondal AK, et al. Endoplasmic reticulum stress markers are associated with obesity in nondiabetic subjects. $J$ Clin Endocrinol Metab. 2008;93:4532-4541. doi:10.1210/jc.20081001

13. Yu X, Ren L-P, Wang C, et al. Role of X-box binding protein-1 in fructose-induced de novo lipogenesis in HepG2 cells. Chin Med J (Engl). 2018;131:2310-2319. doi:10.4103/0366-6999.241799

14. Yilmaz E. Endoplasmic reticulum stress and obesity. Adv Exp Med Biol. 2017;960:261-276. doi:10.1007/978-3-319-48382-5 11
15. Zobel EH, Hansen TW, Rossing P, von Scholten BJ. Global changes in food supply and the obesity epidemic. Curr Obes Rep. 2016;5:449-455. doi:10.1007/s13679-016-0233-8

16. Wang N, Wang J, Tang H-P, et al. Depot-specific inflammation with decreased expression of ATM2 in white adipose tissues induced by high-margarine/lard intake. PLoS One. 2017;12:e0188007. doi:10. 1371/journal.pone.0188007

17. Jeyakumar SM, Vajreswari A, Giridharan NV. Chronic dietary vitamin A supplementation regulates obesity in an obese mutant WNIN/ Ob rat model. Obesity (Silver Spring). 2006;14:52-59. doi:10.1038/ oby. 2006.7

18. Shah SS, Rodriguez G, Musick A, et al. Targeting glioblastoma stem cells with 2-deoxy-D-glucose (2-DG) potentiates radiation-induced Unfolded Protein Response (UPR). Cancers (Basel). 2019;11:159. doi:10.3390/cancers 11020159

19. Zou C, Wang Y, Shen Z. 2-NBDG as a fluorescent indicator for direct glucose uptake measurement. $J$ Biochem Biophys Methods. 2005;64:207-215. doi:10.1016/j.jbbm.2005.08.001

20. Miyamoto JÉ, Ferraz ACG, Portovedo M, et al. Interesterified soybean oil promotes weight gain, impaired glucose tolerance and increased liver cellular stress markers. $J$ Nutr Biochem. 2018;59:153-159. doi:10.1016/j.jnutbio.2018.05.014

21. Hung CS, Lee J-K, Yang C-Y, et al. Measurement of visceral fat: should we include retroperitoneal fat? PLoS One. 2014;9:e112355. doi:10.1371/journal.pone.0112355

22. Zhao X, Shen $\mathrm{C}$, Zhu $\mathrm{H}$, et al. Trans-fatty acids aggravate obesity, insulin resistance and hepatic steatosis in C57BL/6 mice, possibly by suppressing the IRS1 dependent pathway. Molecules. 2016;21:705. doi: $10.3390 /$ molecules21060705

23. Kavanagh K, Jones KL, Sawyer J, et al. Trans fat diet induces abdominal obesity and changes in insulin sensitivity in monkeys. Obesity (Silver Spring). 2007;15:1675-1684. doi:10.1038/oby.2007. 200

24. Brouwer IA, Wanders AJ, Katan MB. Effect of animal and industrial trans fatty acids on HDL and LDL cholesterol levels in humans-a quantitative review. PLoS One. 2010;5:e9434. doi:10.1371/journal. pone.0009434

25. Aronis KN, Joseph RJ, Blackburn GL, Mantzoros C. trans-Fatty acids, insulin resistance/diabetes, and cardiovascular disease risk: should policy decisions be based on observational cohort studies, or should we be waiting for results from randomized placebo-controlled trials? Metabolism. 2011;60:901-905. doi:10.1016/j.metabol.2011.04. 003

26. Hunter JE. Dietary trans fatty acids: review of recent human studies and food industry responses. Lipids. 2006;41:967-992. doi:10.1007/ s11745-006-5049-y

27. Micha R, King IB, Lemaitre RN, et al. Food sources of individual plasma phospholipid trans fatty acid isomers: the cardiovascular health study. Am J Clin Nutr. 2010;91:883-893. doi:10.3945/ ajen.2009.28877

28. Oshita T, Toh R, Shinohara M, et al. Elevated serum elaidic acid predicts risk of repeat revascularization after percutaneous coronary intervention in Japan. Circ J. 2019. doi:10.1253/circj.CJ-18-1175

29. Nishida C, Uauy R, Kumanyika S, Shetty P. The joint WHO/FAO expert consultation on diet, nutrition and the prevention of chronic diseases: process, product and policy implications. Public Health Nutr. 2004;7:245-250.

30. Bendsen NT, Christensen R, Bartels EM, Astrup A. Consumption of industrial and ruminant trans fatty acids and risk of coronary heart disease: a systematic review and meta-analysis of cohort studies. Eur J Clin Nutr. 2011;65:773-783. doi:10.1038/ejen.2011.34

31. Hwang E-Y, Yu M-H, Jung Y-S, Lee S-P, Shon JH, Lee S-O. Defatted safflower seed extract inhibits adipogenesis in 3T3-L1 preadipocytes and improves lipid profiles in C57BL/6J ob/ob mice fed a high-fat diet. Nutr Res. 2016;36:995-1003. doi:10.1016/j.nutres.2016.07.004 
32. Rosenson RS, Brewer HB, Chapman MJ, et al. HDL measures, particle heterogeneity, proposed nomenclature, and relation to atherosclerotic cardiovascular events. Clin Chem. 2011;57:392-410. doi:10.1373/clinchem.2010.155333

33. Kosmas CE, Martinez I, Sourlas A, et al. High-density lipoprotein (HDL) functionality and its relevance to atherosclerotic cardiovascular disease. Drugs Context. 2018;7:212525. doi:10.7573/dic.212525

34. Pagano ES, Spinedi E, Gagliardino JJ. White adipose tissue and circadian rhythm dysfunctions in obesity: pathogenesis and available therapies. Neuroendocrinology. 2017;104:347-363. doi:10.1159/000 453317

35. Sun K, Kusminski CM, Scherer PE. Adipose tissue remodeling and obesity. J Clin Invest. 2011;121:2094-2101. doi:10.1172/JCI45887

36. Wang H, Sit WH, Tipoe GL, Liu Z, Wan JM. Comparative proteomic analysis of fibrotic liver of rats fed high fat diet contained lard versus corn oil. Clin Nutr. 2017;36:198-208. doi:10.1016/j.clnu.2015.10.015

37. Nakandakari S, Muñoz VR, Kuga GK, et al. Short-term high-fat diet modulates several inflammatory, ER stress, and apoptosis markers in the hippocampus of young mice. Brain Behav Immun. 2019. doi:10.1016/j.bbi.2019.02.016

38. Ozcan U, Cao Q, Yilmaz E, et al. Endoplasmic reticulum stress links obesity, insulin action, and type 2 diabetes. Science. 2004;306:457461. doi:10.1126/science. 1103160

39. Chakrabarti A, Chen AW, Varner JD. A review of the mammalian unfolded protein response. Biotechnol Bioeng. 2011;108:2777-2793. doi:10.1002/bit.23282

40. Ibrahim IM, Abdelmalek DH, Elfiky AA. GRP78: A cell's response to stress. Life Sci. 2019. doi:10.1016/j.lfs.2019.04.022

41. Gardner BM, Walter P. Unfolded proteins are Ire1-activating ligands that directly induce the unfolded protein response. Science. 2011;333:1891-1894. doi:10.1126/science.1209126

42. Bertolotti A, Zhang Y, Hendershot LM, Harding HP, Ron D. Dynamic interaction of $\mathrm{BiP}$ and ER stress transducers in the unfolded-protein response. Nat Cell Biol. 2000;2:326-332. doi: $10.1038 / 35014014$

43. Zhang C, Kawauchi J, Adachi MT, et al. Activation of JNK and transcriptional repressor ATF3/LRF1 through the IRE1/TRAF2 pathway is implicated in human vascular endothelial cell death by homocysteine. Biochem Biophys Res Commun. 2001;289:718-724. doi:10.1006/bbrc.2001.6044

44. Shan B, Wang X, Wu Y, et al. The metabolic ER stress sensor IRE1alpha suppresses alternative activation of macrophages and impairs energy expenditure in obesity. Nat Immunol. 2017;18:519529. doi:10.1038/ni.3709

45. Vinayagamoorthi R, Bobby Z, Sridhar MG. Antioxidants preserve redox balance and inhibit c-Jun-N-terminal kinase pathway while improving insulin signaling in fat-fed rats: evidence for the role of oxidative stress on IRS-1 serine phosphorylation and insulin resistance. J Endocrinol. 2008;197:287-296. doi:10.1677/JOE-08-0061

46. Rozo AV, Vijayvargia R, Weiss HR, Ruan H. Silencing Jnk1 and Jnk2 accelerates basal lipolysis and promotes fatty acid re-esterification in mouse adipocytes. Diabetologia. 2008;51:1493-1504. doi:10.1007/ s00125-008-1036-6

47. Ito M, Nagasawa M, Omae N, et al. A novel JNK2/SREBP-1c pathway involved in insulin-induced fatty acid synthesis in human adipocytes. J Lipid Res. 2013;54:1531-1540. doi:10.1194/jlr.M031591

48. Boden G, Merali S. Measurement of the increase in endoplasmic reticulum stress-related proteins and genes in adipose tissue of obese, insulin-resistant individuals. Methods Enzymol. 2011;489:6782. doi:10.1016/B978-0-12-385116-1.00004-2

49. Han J, Kaufman RJ. The role of ER stress in lipid metabolism and lipotoxicity. J Lipid Res. 2016;57:1329-1338. doi:10.1194/jlr.R067 595

50. Welch WJ, Brown CR. Influence of molecular and chemical chaperones on protein folding. Cell Stress Chaperones. 1996;1:109-115. doi:10.1379/1466-1268(1996)001<0109:iomacc >2.3.co;2
51. Haberzettl P, Vladykovskaya E, Srivastava S, Bhatnagar A. Role of endoplasmic reticulum stress in acrolein-induced endothelial activation. Toxicol Appl Pharmacol. 2009;234:14-24. doi:10.1016/j. taap.2008.09.019

52. Inden M, Kitamura Y, Takeuchi H, et al. Neurodegeneration of mouse nigrostriatal dopaminergic system induced by repeated oral administration of rotenone is prevented by 4-phenylbutyrate, a chemical chaperone. J Neurochem. 2007;101:1491-1504. doi:10.1111/j.14714159.2006.04440.x

53. Gould GW, Holman GD. The glucose transporter family: structure, function and tissue-specific expression. Biochem J. 1993;295(Pt 2):329-341. doi:10.1042/bj2950329

54. Bryant NJ, Govers R, James DE. Regulated transport of the glucose transporter GLUT4. Nat Rev Mol Cell Biol. 2002;3:267-277. doi: $10.1038 / \mathrm{nrm} 782$

55. Brewer PD, Habtemichael EN, Romenskaia I, Mastick CC, Coster AC. Glut4 is sorted from a Rab10 GTPase-independent constitutive recycling pathway into a highly insulin-responsive Rab10 GTPasedependent sequestration pathway after adipocyte differentiation. $J$ Biol Chem. 2016;291:773-789. doi:10.1074/jbc.M115.694919

56. Granados N, Amengual J, Ribot J, Palou A, Bonet ML. Distinct effects of oleic acid and its trans-isomer elaidic acid on the expression of myokines and adipokines in cell models. $\mathrm{Br} J$ Nutr. 2011;105:1226-1234. doi:10.1017/S0007114510004885

57. Saravanan N, Haseeb A, Ehtesham NZ; Ghafoorunissa. Differential effects of dietary saturated and trans-fatty acids on expression of genes associated with insulin sensitivity in rat adipose tissue. Eur $J$ Endocrinol. 2005;153:159-165. doi:10.1530/eje.1.01946.

58. Li J, Houseknecht KL, Stenbit AE, Katz EB, Charron MJ. Reduced glucose uptake precedes insulin signaling defects in adipocytes from heterozygous GLUT4 knockout mice. Faseb J. 2000;14:1117-1125. doi:10.1096/fasebj.14.9.1117

59. Ozawa K, Miyazaki M, Matsuhisa M, et al. The endoplasmic reticulum chaperone improves insulin resistance in type 2 diabetes. Diabetes. 2005;54:657-663. doi:10.2337/diabetes.54.3.657

60. Nakatani Y, Kaneto H, Kawamori D, et al. Involvement of endoplasmic reticulum stress in insulin resistance and diabetes. J Biol Chem. 2005;280:847-851. doi:10.1074/jbc.M411860200

61. Guo Q, Xu L, Li H, Sun H, Wu S, Zhou B. 4-PBA reverses autophagic dysfunction and improves insulin sensitivity in adipose tissue of obese mice via Akt/mTOR signaling. Biochem Biophys Res Commun. 2017;484:529-535. doi:10.1016/j.bbrc.2017.01.106

62. Copps KD, White MF. Regulation of insulin sensitivity by serine/threonine phosphorylation of insulin receptor substrate proteins IRS1 and IRS2. Diabetologia. 2012;55:2565-2582. doi:10.1007/s00125-012-2644-8

63. Mahmutovic L, Bego T, Sterner M, et al. Association of IRS1 genetic variants with glucose control and insulin resistance in type 2 diabetic patients from Bosnia and Herzegovina. Drug Metab Pers Ther. 2019;34. doi:10.1515/dmpt-2018-0031.

64. Bluher M, Michael MD, Peroni OD, et al. Adipose tissue selective insulin receptor knockout protects against obesity and obesity-related glucose intolerance. Dev Cell. 2002;3:25-38.

65. Favaretto F, Milan G, Collin GB, et al. GLUT4 defects in adipose tissue are early signs of metabolic alterations in Alms1GT/GT, a mouse model for obesity and insulin resistance. PLoS One. 2014;9: e109540. doi:10.1371/journal.pone.0109540

66. Rondinone CM, Wang LM, Lonnroth P, Wesslau C, Pierce JH, Smith U. Insulin receptor substrate (IRS) 1 is reduced and IRS-2 is the main docking protein for phosphatidylinositol 3-kinase in adipocytes from subjects with non-insulin-dependent diabetes mellitus. Proc Natl Acad Sci U S A. 1997;94:4171-4175. doi:10.1073/pnas.94.8.4171

67. Staubs PA, Nelson JG, Reichart DR, Olefsky JM. Platelet-derived growth factor inhibits insulin stimulation of insulin receptor substrate-1-associated phosphatidylinositol 3-kinase in 3T3-L1 adipocytes without affecting glucose transport. J Biol Chem. 1998; 273:25139-25147. doi:10.1074/jbc.273.39.25139 
68. Chiu TT, Sun Y, Koshkina A, Klip A. Rac-1 superactivation triggers insulin-independent glucose transporter 4 (GLUT4) translocation that bypasses signaling defects exerted by c-Jun $\mathrm{N}$-terminal kinase (JNK)- and ceramide-induced insulin resistance. J Biol Chem. 2013;288:17520-17531. doi:10.1074/jbc.M1 13.467647

69. Brown MS, Goldstein JL. The SREBP pathway: regulation of cholesterol metabolism by proteolysis of a membrane-bound transcription factor. Cell. 1997;89:331-340. doi:10.1016/s0092-8674(00) 80213-5

70. Jang J, Jung Y, Seo SJ, et al. Berberine activates AMPK to suppress proteolytic processing, nuclear translocation and target DNA binding of SREBP-1c in 3T3-L1 adipocytes. Mol Med Rep. 2017;15:41394147. doi: $10.3892 / \mathrm{mmr} .2017 .6513$
71. Zhang C, Chen X, Zhu R-M, et al. Endoplasmic reticulum stress is involved in hepatic SREBP-1c activation and lipid accumulation in fructose-fed mice. Toxicol Lett. 2012;212:229-240. doi:10.1016/j. toxlet.2012.06.002

72. Fang DL, Wan Y, Shen W, et al. Endoplasmic reticulum stress leads to lipid accumulation through upregulation of SREBP-1c in normal hepatic and hepatoma cells. Mol Cell Biochem. 2013;381:127-137. doi:10.1007/s11010-013-1694-7

73. Ning J, Hong T, Ward A, et al. Constitutive role for IRE1alpha-XBP1 signaling pathway in the insulin-mediated hepatic lipogenic program. Endocrinology. 2011;152:2247-2255. doi:10.1210/en.2010-1036

74. Kim JB, Spiegelman BM. ADD1/SREBP1 promotes adipocyte differentiation and gene expression linked to fatty acid metabolism. Genes Dev. 1996;10:1096-1107. doi:10.1101/gad.10.9.1096

Diabetes, Metabolic Syndrome and Obesity: Targets and Therapy

\section{Publish your work in this journal}

Diabetes, Metabolic Syndrome and Obesity: Targets and Therapy is an international, peer-reviewed open-access journal committed to the rapid publication of the latest laboratory and clinical findings in the fields of diabetes, metabolic syndrome and obesity research. Original research, review, case reports, hypothesis formation, expert opinion and commentaries are all considered for publication. The manuscript management system is completely online and includes a very quick and fair peer-review system, which is all easy to use. Visit http://www.dovepress.com/testimonials.php to read real quotes from published authors.

Submit your manuscript here: https://www.dovepress.com/diabetes-metabolic-syndrome-and-obesity-targets-and-therapy-journal 